\title{
Manejo del drenaje aspirativo en dermolipectomía abdominal en pacientes postbariátricos
}

\section{Aspirative drainage system management in ahdominoplasty in post-bariatric patients}

\author{
Linda L. RINCÓN-RUBIO*, Marisela CEMBORAIN-VALARINO**, Katiana U. GUTIÉRREZ-BARROZO***, \\ Bernardette G. GIL-MASROUA****, Angelique M. BOOKAMAN-SALAZAR ${ }^{\star \star * * *}$
}

Resumen

Antecedentes y Objetivo. En los pacientes con pérdida masiva de peso, la formación de seroma tras dermolipectomía abdominal es una complicación frecuente. Nos planteamos describir el manejo del drenaje aspirativo en estos pacientes considerando los factores predisponentes para la formación de seroma, y determinar si el área de disección de la dermolipectomía abdominal en pacientes postbariátricos, y su condición metabólica aumenta el volumen obtenido a través de los drenajes aspirativos, así como el tiempo hasta la retirada de los mismos.

Material y Método. Realizamos un estudio clínico observacional, descriptivo, prospectivo, de corte longitudinal. La muestra consistió en 30 pacientes a los que se les realizó dermolipectomía abdominal postbariátrica, se midió el área de disección intraoperatoria y se registró el volumen del gasto del drenaje hasta su retirada.

Resultados. El $83.3 \%$ de los pacientes fueron mujeres, con edad meda de $38.9+10.9$ años e índice de masa corporal de 27.85 $+5.49 \mathrm{~kg} / \mathrm{m}^{2}$. El promedio del área de disección fue de $582.983+$ $173.90 \mathrm{~cm}^{2}$, mientras que el volumen diario obtenido por el drenaje fue de $72.12+24.19 \mathrm{ml}$. Retiramos el drenaje con mayor frecuencia entre los días 9 y 10 de postoperatorio.

Conclusiones. La condición postbariátrica, con gran área de disección en la dermolipectomía abdominal, está relacionada con una mayor producción de líquido en el postoperatorio, por lo cual se deben cambiar los parámetros comúnmente empleados para la retirada de los drenajes.
Background and Objective. A common complication after abdominoplasty in patients with massive weight loss is seroma formation. This condition could change the traditional parameters to remove drainage systems. Our objective are to determine if the post-bariatric condition is a factor that decreases the absorption capacity of fluid in the body surface in dermolipectomy, to know the relationship between the dissected area with the amount of surface drainage, to consider if the average of drainage in 24 hours may be the highest standard for removing the drain, and to describe the biochemical parameters related to the volume of the drainage

Methods. We conduct a prospective, longitudinal, descriptive and clinical study with a sample consisted on 30 post-bariatric patients who underwent abdominal dermolipectomy. The intraoperative dissection area was measured and the volume of drainage registered until its removal.

Results. The mean age was $38.9+10.9$ years with $83.3 \%$ female The average body mass index was $27.85+5.49 \mathrm{~kg} / \mathrm{m}^{2}$. The average area of dissection was $582.983+173.90 \mathrm{~cm}^{2}$. Drain removal was conducted between 9 and 10 postoperative days. The daily volume obtained by drainage was $72.12+24.19 \mathrm{ml}$.

Conclusions. The postbariatric condition of the patients, with large areas of dissection in abdominal dermolipectomies, is related to greater production of fluid in the postoperative period, which is the reason why the parameters that are commonly used for drain removal must be changed.

\section{Palabras clave Cirugía bariátrica, Cirugía postbariatrica, Abdominoplastia. Drenajes.}

Nivel de evidencia científica

Recibido [esta versión]

Aceptado
$4 c$ Terapéutico

19 febrero/2018

$5 \mathrm{marzo} / 2018$

\section{Key words Bariatric surgery, Post-bariatric sugery, Abdominoplasty, Drainages.}

Level of evidence

Received [this version] Accepted
$4 \mathrm{c}$ Therapeutic

19 february/2018 $5 \mathrm{march} / 2018$

Conflicto de intereses: los autores declaran no tener ningún interés financiero relacionado con el contenido de este artículo.

* Especialista en Cirugía Plástica, Reconstructiva y Maxilofacial, Directora de la Unidad de Cirugía Plástica y Postbariátrica Caracas, Profesora Universitaria en el Hospital Carlos J. Bello Cruz Roja Venezolana, Especialista en el Centro Diagnóstico Docente Las Mercedes, Caracas, Venezuela.

** Especialista en Cirugía Plástica, Reconstructiva y Maxilofacial, Co-Directora de la Unidad de Cirugía Plástica y Postbariátrica Caracas, Especialista en la Clínica Urológico San Román, Caracas, Venezuela.

*** Especialista en Cirugía Plástica, Reconstructiva y Maxilofacial, Miembro de la Unidad de Cirugía Plástica y Postbariátrica Caracas-Venezuela. Especialista en el Instituto Policlínico de Turmero, Estado Aragua, Venezuela.

**** Especialista en Cirugía Plástica, Reconstructiva y Maxilofacial, Profesora Universitaria en el Hospital Clínico Universitario de Caracas, Caracas, Venezuela.

${ }^{\star * \star \star \star}$ Especialista en Cirugía Plástica, Reconstructiva y Maxilofacial. Profesora Universitaria en el Hospital Carlos J. Bello Cruz Roja Venezolana, Caracas, Venezuela. 


\section{Introducción}

A finales del siglo XIX, la cirugía de la pared abdominal estaba sujeta a resecciones pequeñas y correcciones de hernias. En muchos casos, la dermolipectomía abdominal o abdominoplastia facilitaba la herniorrafia a la vez que la opción de resección del exceso de tejido dermograso. Con la evolución de las técnicas de realización de abdominoplastia llegando al decolamiento del colgajo hasta el reborde costal, la plicatura muscular, el reposicionamiento de la cicatriz umbilical y la resección del exceso del tejido cutáneo, se documentaron complicaciones frecuentes como hematomas y seromas, con presencia de líquido que puede comprometer la cicatrización y propiciar la aparición de infecciones.

Es por todo ello que Vinicius y col., ${ }^{(1)}$ en 2002, presentan una investigación en la que evalúan la presencia de fluido abdominal tras la realización de dermolipectomías usando drenaje pasivo, drenaje activo y sin drenaje, observando que el uso de drenajes aspirativos activos en dermolipectomías es necesario para disminuir la tasa de producción de seroma y que la producción del mismo no depende de una sola causa, sino que los decolamientos amplios, la hemostasia insuficiente, el uso abusivo del electrocauterio y la compresión abdominal inadecuada, son factores predisponentes para la formación de colecciones serosas.

Posteriormente, Andrades y col. en 2007,(2) realizan un estudio para evaluar la capacidad de la tensión progresiva con suturas para la reducción del seroma postabdominoplastia en comparación con el uso de drenajes convencionales, concluyendo que el uso de puntos de tensión progresiva, a pesar de que reduce el gasto a través del drenaje, incrementa el tiempo quirúrgico y presenta la misma incidencia de seromas que cuando se usan solamente drenajes, hecho comprobado por evaluación clínica y por ecografía; es por esto que la combinación de ambos métodos de forma simultánea no parece tener ninguna ventaja, pero si no se usa ningún método, las complicaciones son mayores. Así mismo Borile y col. ${ }^{(3)}$ en 2008 , realizan dermolipectomía abdominal en un grupo de pacientes después del primer embarazo, multíparas o que perdieron recientemente peso, contrastando el uso o no de drenajes con presión negativa y colocación de faja compresiva en el postoperatorio, concluyendo que el uso de drenajes durante un largo período de tiempo aumenta el disconfort y reduce la movilidad del paciente, contribuyendo además al aumento de la incidencia de morbilidades asociadas, como infección local y eventos tromboembólicos.

En ese orden de ideas, Fang y col., ${ }^{(4)}$ en 2010, afirman que realizar la dermolipectomía en un plano más superficial, disminuye la necesidad de uso de drenajes en cuanto al número de días. Este hallazgo se ha asociado a la preservación de la fascia de Scarpa en las dermolipectomías convencionales, así como de los vasos linfáticos abdominales. En base a ello, Costa-Ferreira y col., en 2010 y $2013,{ }^{(5,6)}$ publican sus investigaciones en las que dividen la muestra en 2 grupos; a uno se le realizó una abdominoplastia clásica y al otro una abdominoplastia con preservación de la fascia de Scarpa infraumbilical, concluyendo que la preservación de la misma reduce tanto la formación de seromas como el volumen de gasto del drenaje y el tiempo para su retirada en el postoperatorio.

En 2015, Friedman y col..$^{(7)}$ caracterizaron la arquitectura linfática de la pared abdominal y aportaron un concepto básico para poder refinar las técnicas de abdominoplastia. Analizaron 15 muestras de tejido de pacientes sometidos a dermolipectomía, teñidas con hematoxilina y eosina e inmunohistoquímica, en las que se usaron $\mathrm{CD} 31$ y D2-40 para determinar el número de linfáticos. Esto les permitió concluir que los linfáticos se encuentran significativamente localizados en la dermis, pero también están presentes en las capas cercanas a la fascia, confirmando la presencia de canales linfáticos profundos que podrían potencialmente ser preservados durante una abdominoplastia. Sin embargo, debido al curso incierto de los vasos linfáticos colectores cutáneos en la pared abdominal en relación con la fascia de Scarpa, Tourani y col., en 2015, ${ }^{(8)}$ realizaron un estudio en cadáveres en el que plantearon que la preservación de la fascia de Scarpa en la región baja abdominal se asocia a la reducción de la tasa de formación de seromas en las abdominoplastias dado que los linfáticos de la región baja del abdomen corren en profundidad a lo largo de esta capa. Concluyeron que si se preserva la fascia de Scarpa se podrían preservar los vasos colectores de la pared abdominal. Basándose en esta presunción, muchos cirujanos comenzaron a combinar la liposucción abdominal con dermolipectomía para reducir el área de disección y provacar un menor daño a la fascia, con lo que se lograría la disminución de los seromas postoperatorios. Asi lo plantean Moretti y col. en 2006 $6^{(9)}$ estudiando la incidencia de formación de seromas como complicación de mayor frecuencia en las dermolipectomías, tanto desde el punto de vista experimental como histopatológico y clínico en pacientes sometidos a liposucción abdominal, dermolipectomía, y ambos procedimientos combinados, concluyendo que la cirugía combinada es la que presenta mayor incidencia de formación de seromas.

Por su parte, Llanos y col., en 2013, ${ }^{(10)}$ investigan la incidencia de seromas en pacientes sometidos a dermolipectomía abdominal asociada a lipoaspiración, incluyendo o no la reparación de la fascia de Scarpa y determinando el resultado estético del procedimiento, concluyendo que la reparación de la dicha fascia no conlleva una repercusión negativa desde el punto de vista estético y sin embargo disminuye la incidencia de formación de seromas.

En vista de que existen múltiples estudios que intentan demostrar los factores que influyen en la formación de seromas tras la realización de una abdominoplastia, y por ende la necesidad del uso de drenajes así como la inclusión de suturas de tensión progresiva o la preservación de la fascia de Scarpa, e incluso la combinación con 
liposucción, Quaba y col., ${ }^{(1)}$ en 2015, estudian de manera retrospectiva, entre 2003 y 2012, aquellos pacientes a los que habían realizado dermolipectomía sin uso de drenajes ni de suturas de tensión progresiva. Concluyen que se pueden realizar dermolipectomías de manera segura sin ningún tipo de drenaje o sutura. También Di Martino y col., ${ }^{(12)}$ realizan un estudio en 2015 para describir la historia natural de la formación de seromas tras abdominoplastia, definiendo el seroma cuando se evidencia un volumen mayor de $20 \mathrm{ml}$ de fluido y concluyendo que la incidencia más alta de seroma se produjo entre sus pacientes en el día 11 de postoperatorio.

Todos estos hallazgos resultan de interés debido a que en la práctica habitual del cirujano plástico en dermolipectomía abdominal en pacientes no postbariátricos, se estima que un promedio de 7 días de mantenimiento del drenaje aspirativo, asociado a un promedio de $30 \mathrm{ml}$ de gasto en 24 horas, es el indicador para la retirada de los drenajes.

Sin embargo, existe una población especial que debemos considerar. Cuando los pacientes han sido sometidos a procedimientos quirúrgicos o regímenes nutricionales para la disminución de peso logrando una pérdida masiva del mismo, suelen tener como resultado una hiperflacidez del estrato dermograso que tiene como consecuencia el que muchos de ellos consulten al cirujano plástico en búsqueda de una solución a su problema. La superficie corporal de los pacientes con sobrepeso tiende a ser más amplia que la de la población normal, explicándose este fenómeno por el aumento de las dimensiones músculo-esqueléticas en compensación al peso, es decir, las estructuras se adaptan para soportar determinado peso. Por esta razón es común observar en pacientes postpérdida masiva de peso que las superficies disecadas durante la dermolipectomía son también más amplias. Aunado a esto, estos pacientes presentan una condición postbariátrica que afecta en cantidad y calidad a los diferentes componentes del estrato dermograso y a sus características bioquímicas, asociándose en ellos índices más altos de formación de seroma postoperatorio.

Shermak y col. ${ }^{(13)}$ en 2008, realizan una investigación en la que describen que las complicaciones más comunes en la cirugía del contorno corporal en pacientes con pérdida masiva de peso son la cicatrización retardada y el seroma. De todos los procedimientos, el $14 \%$ de los pacientes presentó seroma y de ellos el $12 \%$ eran aquellos a los que se les había realizado una abdominoplastia. El mayor riesgo encontrado para la formación de seroma fue el peso del colgajo escindido, aumentando el riesgo en un $9 \%$ por cada libra de piel retirada.

En nuestra población de pacientes postpérdida de peso masiva y sometidos a distintas técnicas de dermolipectomía abdominal, observamos un gasto continuo por el drenaje que habitualmente sobrepasa los 7 días de postoperatorio, con un promedio de gasto más alto que en los pacientes sin pérdida masiva de peso. Esto nos llevó a plantear si existe alguna relación con la condición post- bariátrica de los mismos o con la extensión del área quirúrgica que nos permitiera modificar los parámetros que se vienen usando hasta la actualidad para decidir el momento de retirada de los drenajes.

La presente investigación está dirigida a describir el manejo de los drenajes aspirativos en los pacientes postpérdida masiva de peso sometidos a abdominoplastia y su relación con los parámetros metabólicos propios de estos pacientes, así como el área de disección en el momento de realizar la dermolipectomía. Para ello, resulta conveniente describir las características clínicas de dichos pacientes, sus parámetros metabólicos, así como categorizar los procedimientos quirúrgicos realizados, determinar el área de disección y el volumen de líquido obtenido hasta la retirada de los drenajes, y establecer la cantidad en mililitros de trasudado obtenido por área en $\mathrm{cm}^{2}$ disecada. Finalmente, establecer si existe alguna relación entre el volumen de drenaje y el área de disección o los parámetros metabólicos, que nos permita definir un protocolo de manejo del drenaje aspirativo en la dermolipectomía abdominal en pacientes postbariátricos

Seguimos así la siguiente hipótesis: el área de disección de la dermolipectomía abdominal en pacientes postbariátricos, así como su condición metabólica, aumenta el volumen obtenido a través de los drenajes aspirativos y a su vez el tiempo hasta la retirada de los mismos en el postoperatorio.

\section{Material y método}

Realizamos un estudio clínico, observacional, descriptivo, prospectivo de corte longitudinal, cuya población estuvo integrada por 42 pacientes que acudieron a la Unidad de Cirugía Plástica y Postbariátrica de Caracas, Venezuela, y cuyo principal motivo de consulta fue la mejoría de su contorno corporal inferior, en un período de 8 meses. De entre todos ellos, seleccionamos una muestra de 30 pacientes a quienes se les realizó dermolipectomía abdominal y que cumplían unos criterios de inclusión y exclusión establecidos (Tabla I).

Tabla I. Criterios de inclusión y exclusión

\begin{tabular}{|c|c|}
\hline Criterios de inclusión & Criterios de exclusión \\
\hline $\begin{array}{l}\text { - Mínimo } 1 \text { año después de ci- } \\
\text { rugía bariátrica. } \\
\text { - Peso estable por } 3 \text { meses. } \\
\text { - Meta de pérdida de peso al- } \\
\text { canzada }>60 \% \text {. } \\
\text { - Enfermedades asociadas con- } \\
\text { troladas. } \\
\text { - Referencia de cirujano bariá- } \\
\text { trico. } \\
\text { - Mayores de } 18 \text { años y meno- } \\
\text { res de } 65 \text { años. } \\
\text { - Cumplimiento del protocolo } \\
\text { preoperatiro. } \\
\text { - Firma del consentimiento in- } \\
\text { formado. }\end{array}$ & $\begin{array}{l}\text { - } \text { IMC }>35 \text {. } \\
\text { labosultados de exámenes de } \\
\text { - Menores de } 18 \text { años y mayo- } \\
\text { res de } 65 \text { años. }\end{array}$ \\
\hline
\end{tabular}


Obtuvimos de las historias clínicas los datos concernientes a las características clínicas, parámetros metabólicos, así como los procedimientos quirúrgicos realizados en el grupo de pacientes seleccionados (sexo, edad, proteínas totales, albúmina, globulina, hemoglobina, índice de masa corporal-IMC y tipo de cirugía a realizar).

Expresamos las variables de proporción en media y desviación típica, y las variables nominales en frecuencia. Empleamos el manejo estadístico con media, desviación típica, error típico de la media y significancia para establecer la correlación entre la variable de volumen diario de líquido y los niveles de albúmina; y el coeficiente de Pearson para establecer la relación entre el área de disección y el volumen inicial, el volumen de retirada y el promedio diario de líquido, utilizando el programa Microsof Excel ${ }^{\circledR}$ para Windows 7.

Durante la intervención quirúrgica llevamos a cabo distintas técnicas de dermolipectomía abdominal para pacientes con pérdida masiva de peso. La disección de los colgajos se realizó en un plano superior a la fascia de Scarpa empleando puntas de electro-bisturí no refractarias, con cubierta de cerámica no conductiva (Epitome scalpel, Utah Medical Prductis Inc. EE.UU. Para la medición del área de disección diseñamos un modelo geométrico formado por 1 cuadrado y 3 triángulos (Fig. 1). Después resecamos el colgajo dermograso mediante técnica dinámica y segmentaria, no en bloque, y cerramos la herida con sutura no absorbible en 3 planos, sin puntos de aproximación del colgajo a la pared abdominal.

Colocamos en todos los pacientes un drenaje cerrado aspirativo de silicona con exteriorización en el pubis por contraabertura. En los casos en que la dermolipectomía tuvo un componente vertical, colocamos un segundo drenaje dirigido hacia el área de disección de la línea media abdominal, que de igual manera, exteriorizamos en el pubis por contraabertura.

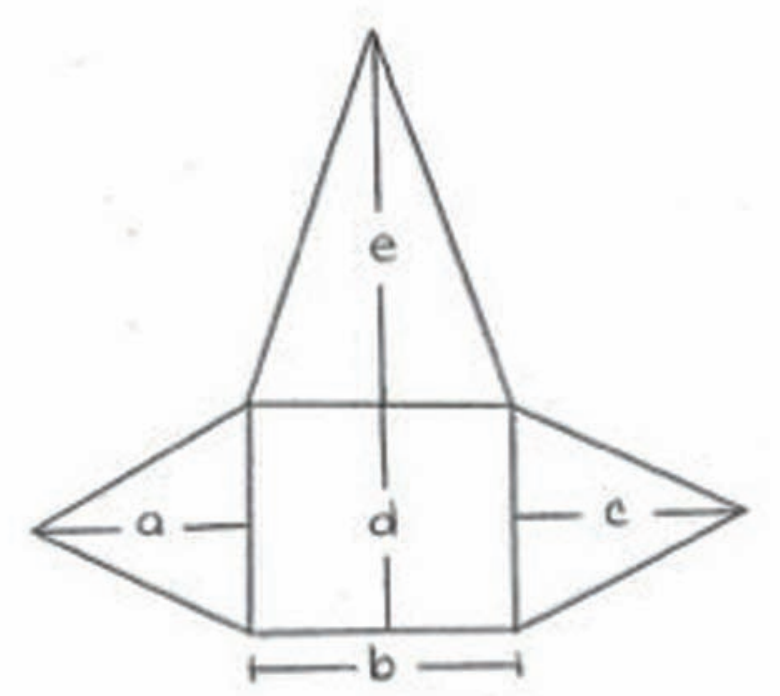

Fig. 1. Modelo geométrico, para calcular el área de disección intraoperatoria Área total: área cuadrado + área triángulo 1 + área triángulo 2 + área triángulo 3.

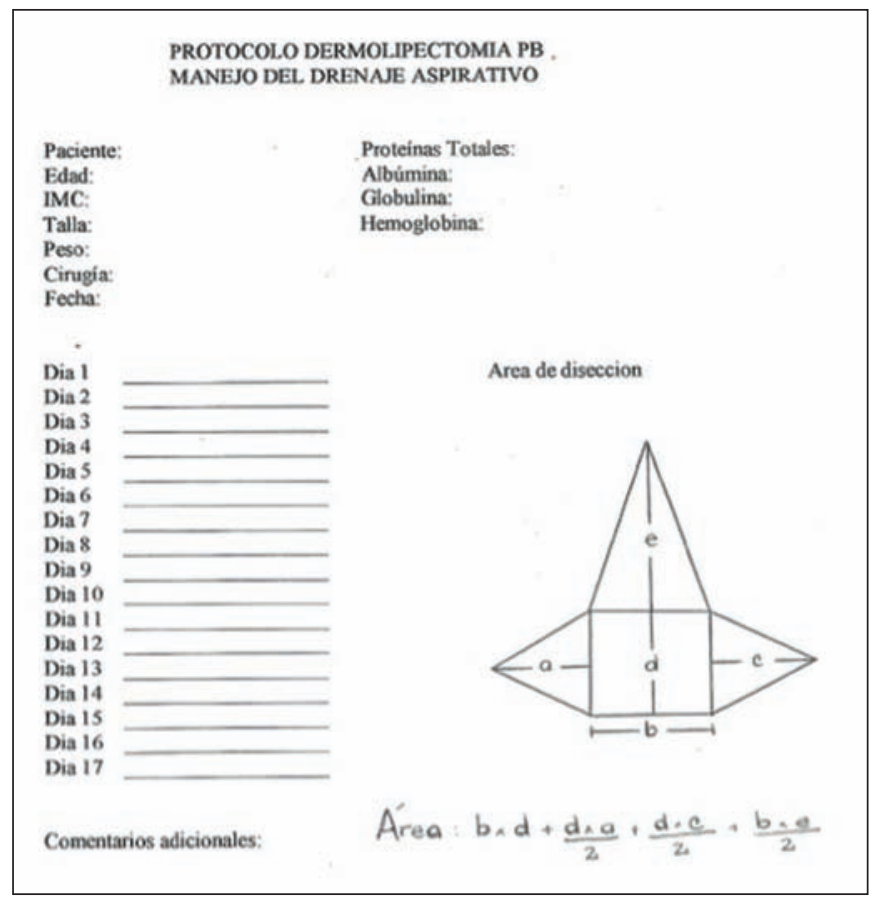

Fig. 2. Ficha de recolección de datos. Modelo geométrico para calcular el área de disección intraoperatoria.

Durante el postoperatorio registramos en una ficha el volumen diario de gasto a través de los drenajes, así como las medidas del área de disección (Fig. 2). Para la retirada de los drenajes establecimos como punto de referencia que el gasto a través de los mismos en 24 horas se mantuviera sin variación ascendente y con el mismo volumen durante 48 horas consecutivas después del $7^{\circ}$ día de postoperatorio.

En los casos en los que observamos un aumento brusco en el volumen de gasto a través del drenaje cuando ya venía en descenso a los 7 días, llevamos a cabo un pinzamiento de la manguera del drenaje mediante pinza de Crille con la finalidad de detener la aspiración, y procedimos a realizar un estudio mediante ultrasonido de partes blandas (Equipo Logiq $5^{\circledR}$, General Electric Company, EE.UU.) con transductor lineal de $10 \mathrm{MHz}$ y para músculo esquelético, al momento del pinzamiento y a las 24 horas del mismo, evaluando así si el organismo era capaz de absorber el trasudado y, en caso de que demostrar descenso del volumen ecográfico, proceder a la retirada del drenaje.

Todos los pacientes utilizaron prendas de presión abdominal (no tubulares) desde el segundo día de postoperatorio y durante 3 meses, haciéndoles ajustes a medida que el proceso de inflamación mejoraba. Asimismo, usaron un dispositivo artesanal a modo de tabla de presión, hecha con cartón y gomaespuma, para mejorar la coaptación del colgajo abdominal (Fig. 3).

\section{RESULTADOS}

Del total de los 30 pacientes incluidos en el protocolo de investigación, $25(83.3 \%)$ fueron mujeres y $5(16.6 \%)$ varones (Gráfico 1), con una edad promedio de $38.9+$ 10.9 años (rango de 64 a 21 años) y un IMC de $27.8+5.4$ como promedio (Tabla II). En cuanto a los parámetros 

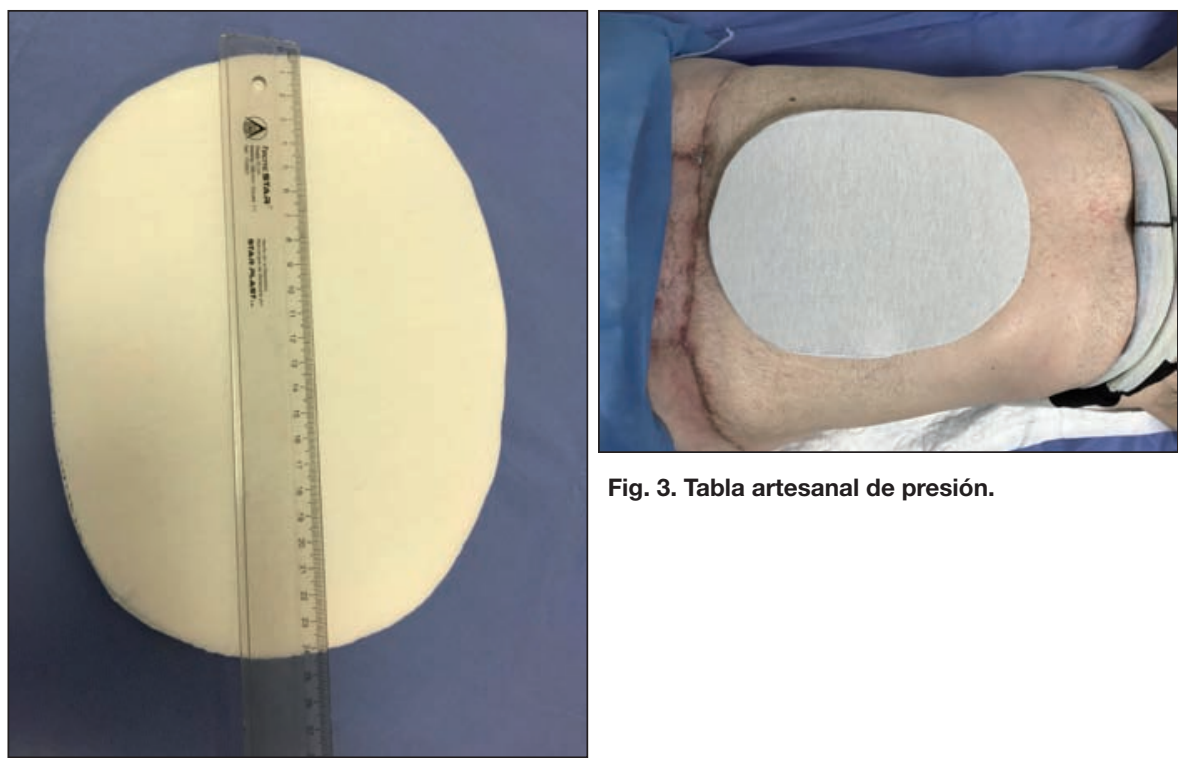

Fig. 3. Tabla artesanal de presión.

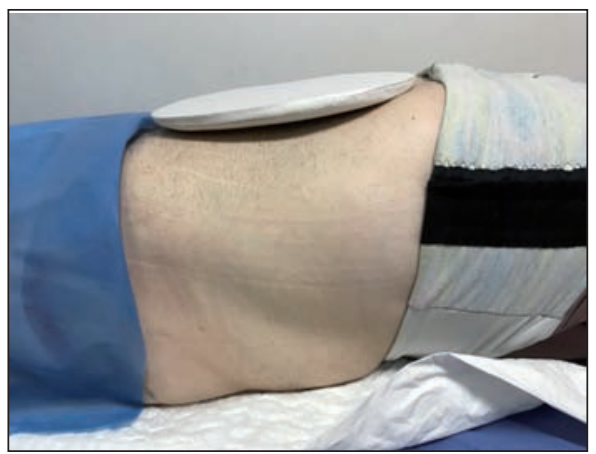

Tabla III. Parámetros metabólicos de los pacientes. ProtTot: proteinas totales. Alb: albúmina. Glob: globulinas. HB: hemoglobina.

\begin{tabular}{|l|l|c|c|c|c|}
\hline \multicolumn{2}{|l|}{} & $\begin{array}{c}\text { ProtTot } \\
(\mathrm{g} / \mathbf{d L})\end{array}$ & $\begin{array}{c}\text { Alb } \\
(\mathbf{g 7 d L})\end{array}$ & $\begin{array}{c}\text { Glob } \\
(\mathbf{g} / \mathbf{d L})\end{array}$ & $\begin{array}{c}\text { HB } \\
(\mathrm{g} / \mathrm{L})\end{array}$ \\
\hline \multirow{2}{*}{$\mathbf{N}$} & Válidos & 30 & 30 & 30 & 30 \\
\cline { 2 - 6 } & Perdidos & 0 & 0 & 0 & 0 \\
\hline Media & 7.0097 & 3.994333 & 3.023000 & 13.173 \\
\hline Desviación típica & 0.39475 & 0.2773108 & 0.4415345 & 0.9384 \\
\hline Mínimo & 6.20 & 3.2000 & 2.1000 & 11.8 \\
\hline Máximo & 7.70 & 4.6300 & 3.9000 & 14.9 \\
\hline
\end{tabular}

Tabla IV. Parámetros metabólicos de los pacientes.

\begin{tabular}{|l|l|c|c|c|c|}
\hline \multicolumn{2}{|l|}{} & $\begin{array}{c}\text { Edad } \\
(\mathbf{a n ̃ o s})\end{array}$ & $\begin{array}{c}\text { IMC } \\
\left(\mathbf{K g} / \mathbf{m}^{2}\right)\end{array}$ & $\begin{array}{c}\text { Peso } \\
(\mathbf{K g})\end{array}$ & $\begin{array}{c}\text { Estatura } \\
(\mathbf{m})\end{array}$ \\
\hline \multirow{2}{*}{$\mathbf{N}$} & Válidos & 30 & 30 & 30 & 30 \\
\cline { 2 - 6 } & Perdidos & 0 & 0 & 0 & 0 \\
\hline Media & 38.90 & 27.853 & 74.027 & 1.6310 \\
\hline \multicolumn{2}{|l|}{ Desviación típica } & 10.921 & 5.4935 & 17.1759 & 0.08568 \\
\hline \multicolumn{2}{|l|}{ Mínimo } & 21 & 20.0 & 54.0 & 1.47 \\
\hline \multicolumn{2}{|l|}{ Máximo } & 64 & 53.0 & 136.0 & 1.82 \\
\hline
\end{tabular}

metabólicos (Tabla III), dentro del protocolo de nuestra Unidad de Cirugía Plástica y Postbariátrica, todos los pacientes deben tener valores de laboratorio con niveles normales; y ya que se ha relacionado la hipoalbuminemia con un mayor gasto a través del drenaje, resaltamos que en estos pacientes, 17 (56.7\%) de la muestra tenían un nivel de albumina sérica por encima de $4 \mathrm{gr} / \mathrm{dl}$ (rango entre 3.2 y $4.6 \mathrm{gr} / \mathrm{dl})$. Sin embargo, esta investigación empleó un

\begin{tabular}{|l|c|c|c|c|c|c|}
\hline & Alb & $\mathbf{N}$ & Media & $\begin{array}{c}\text { Desviación } \\
\text { típica }\end{array}$ & $\begin{array}{c}\text { Error tip. } \\
\text { de la } \\
\text { media }\end{array}$ & Significancia \\
\hline \multirow{2}{*}{$\begin{array}{l}\text { Promedio } \\
\text { diario }\end{array}$} & $>=4.0000$ & 17 & 68.566416 & 24.8303346 & 6.0222407 & $p .0 .366$ \\
\cline { 2 - 7 } & $<4.0000$ & 13 & 76.781724 & 23.4684041 & 6.5089642 & \\
\hline
\end{tabular}

valor óptimo de albúmina de $4 \mathrm{~g} / \mathrm{dl}$ para agrupar a los pacientes y correlacionarlos con el volumen de líquido obtenido a través del drenaje aspirativo en 24 horas, encontrando que el grupo con valores de albúmina por debajo de $4 \mathrm{gr} / \mathrm{dl}$ tenían un mayor promedio de drenaje en la muestra estudiada (76.78 ml/día), diferencia que no fue estadísticamente significativa (p: 0.366) (Tabla IV).

En cuanto al procedimiento quirúrgico realizado, en 19 pacientes (63.3\%) llevamos a cabo una dermolipectomía horizontal (Gráfico 2) en la que el área de disección tuvo una media de $582.98+173.90 \mathrm{~cm}^{2}$ (rango entre $912 \mathrm{~cm}^{2}$ y $315.5 \mathrm{~cm}^{2}$ ) (Gráfico 3).

El volumen a través del drenaje en las primeras 24 horas tuvo un promedio de $108.16 \mathrm{ml}+49.26$; mientras

\section{Alb: albúmina. Glob: globulinas.}


Gráfico 2. Tipo de cirugía ( $\mathrm{N}=30)$

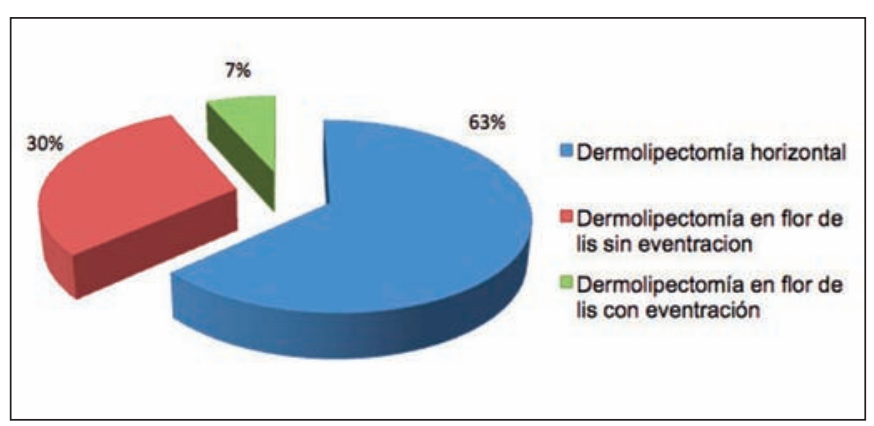

Gráfico 3. Área de disección por paciente ( $\mathrm{N}=30)$

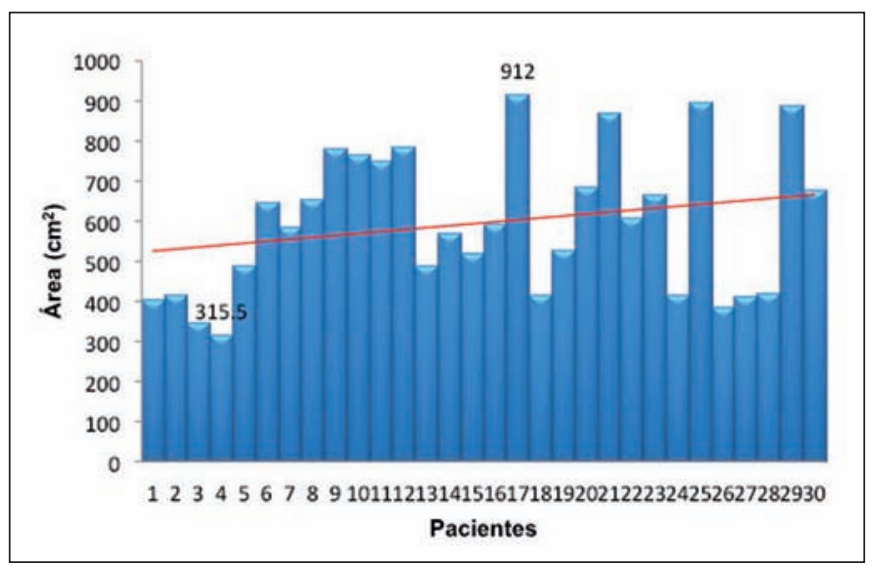

Tabla V. Volumen obtenido a través del drenaje

\begin{tabular}{|l|l|c|c|c|c|}
\hline \multicolumn{2}{|l|}{} & $\begin{array}{c}\text { Volmen } \\
\text { inicial } \\
(\mathbf{m l})\end{array}$ & $\begin{array}{c}\text { Volumen } \\
\text { de retiro } \\
(\mathbf{m l})\end{array}$ & $\begin{array}{c}\text { Promedio } \\
\text { diario } \\
(\mathbf{m l})\end{array}$ & $\begin{array}{c}\text { Volumen } \\
\text { superficie } \\
\left(\mathbf{m l} / \mathbf{c m}^{2}\right)\end{array}$ \\
\hline \multirow{2}{*}{ N } & Válidos & 30 & 30 & 30 & 30 \\
\cline { 2 - 6 } Perdidos & 0 & 0 & 0 & 0 \\
\hline Media & 108.16 & 45.16 & 72.12 & 0.12 \\
\hline Desviación típica & 49.26 & 23.21 & 24.19 & 0.04 \\
\hline Mínimo & 30 & 20 & 30.90 & 0.05 \\
\hline Máximo & 230 & 150 & 148.50 & 0.20 \\
\hline
\end{tabular}

que el volumen diario promedio fue de $72.12+24.19 \mathrm{ml}$ (entre 148.5 y $30.9 \mathrm{ml}$ ) (Tabla V).

Retiramos el sistema de drenaje el día 10 de postoperatorio en 10 pacientes $(33.3 \%)$, en $6(20 \%)$ el día 9 y solo 1 paciente mantuvo el drenaje durante 17 días (Gráfico 4), siendo el promedio de volumen de retirada de $45.16+23.21 \mathrm{ml}$ (rango entre 150 y $20 \mathrm{ml})$ (Tabla V).

Durante el periodo de observación, 3 pacientes presentaron un aumento de volumen a través del drenaje después de ir en descenso, por lo que procedimos a pinzar los drenajes durante 24 horas y realizamos ultrasonido de partes blandas para cuantificar la cantidad de líquido coleccionado en la pared abdominal (Figura 4). A las 24 horas repetimos el uiltrasonido, evidenciando la
Gráfico 4. Distribución según día de retirada del drenaje $(\mathrm{N}=30)$
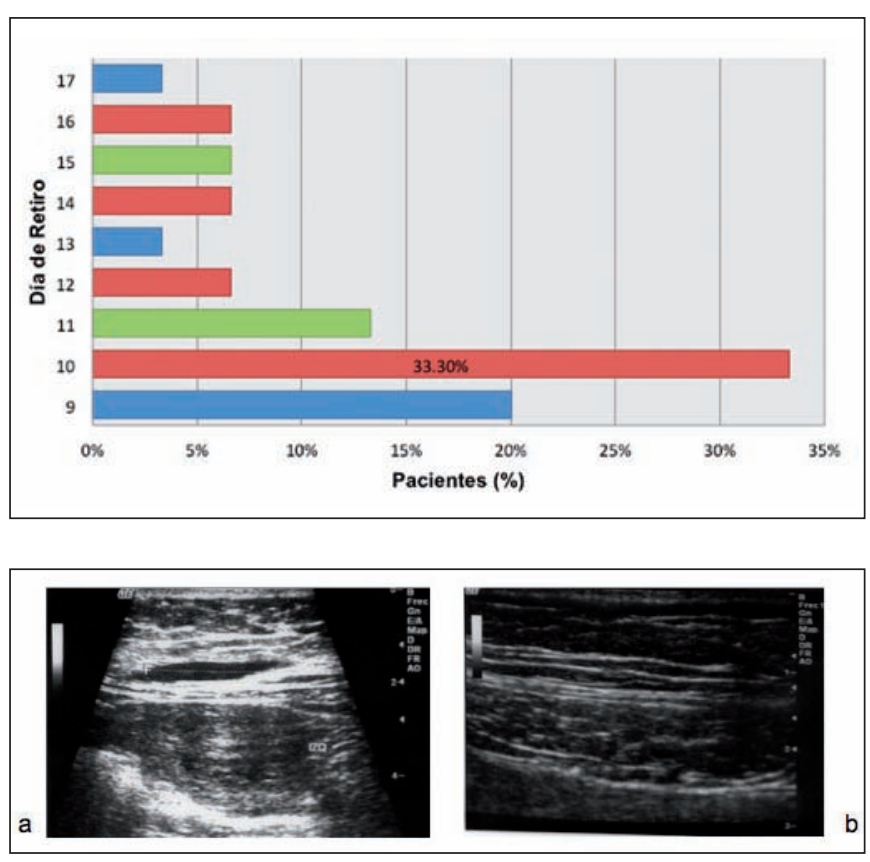

Fig. 4. Varón de 30 años de edad sometido a dermolipectomía abdominal horizontal. Área de disección: $518 \mathrm{~cm}^{2}$, albumina sérica: $4.65 \mathrm{mg} / \mathrm{dl}$. Aumento del gasto a través del drenaje posterior al $9^{\circ}$ día de postoperatorio. A. Imagen de ultrasonido de pared abdominal el día del pinzamiento del drenaje con evidencia de colección de líquido. B. Control ecográfico 24 horas después, cuando se retiró el drenaje.

Gráfico 5. Correlación entre el área de disección y el volumen inicial.

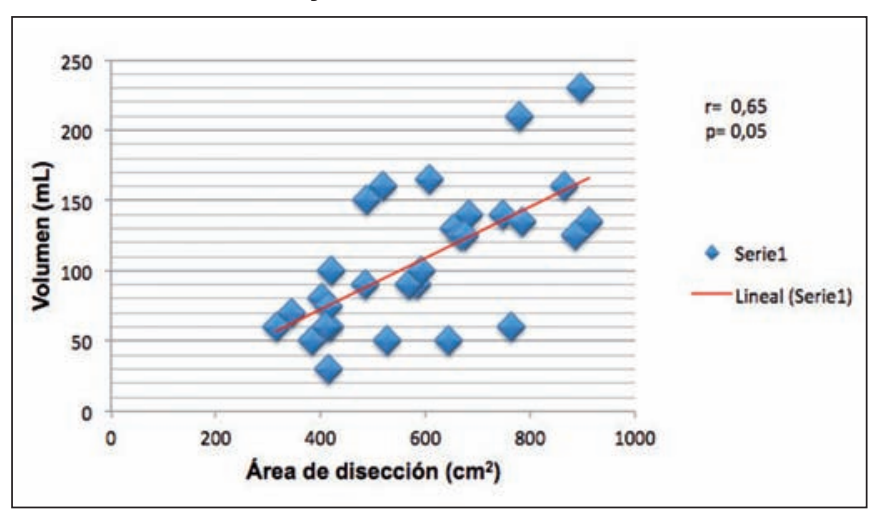

$r$ : Coeficiente de Pearson.

disminución de la colección, por lo que indicamos su retirada.

Con estos hallazgos podemos inferir que $1 \mathrm{~cm}^{2}$ de superficie disecada produce $0.12+0.41 \mathrm{ml}$ en 24 horas, con una correlación lineal de $\mathrm{r}=0.53$ y $\mathrm{p}=0.05$. Asimismo, al comparar el área de disección con el promedio diario de líquido, volumen inicial y volumen con el cual se retiró el drenaje, podemos evidenciar una correlación lineal moderada pero estadísticamente significativa $(\mathrm{p}=0.05)$ en ambos casos (Gráficos 5-7).

\section{Discusión}

El uso de drenajes tras dermolipectomía abdominal en pacientes con antecedente de pérdida masiva de peso o sin esta condición, constituye una práctica habitual 
Gráfico 6. Correlación entre el área de disección y el volumen en el momento de la retirada del drenaje

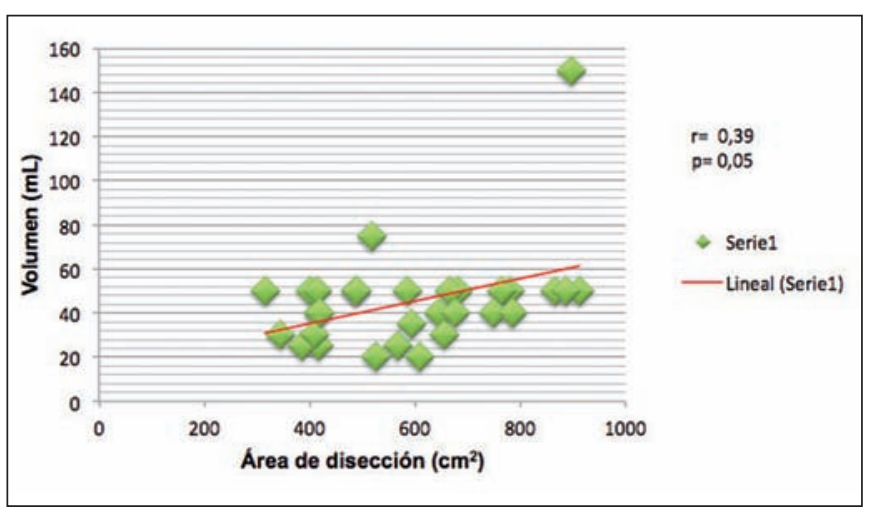

$r$ : Coeficiente de Pearson.

Gráfico 7. Correlación entre el área de disección y el promedio de volumen diario del drenaje

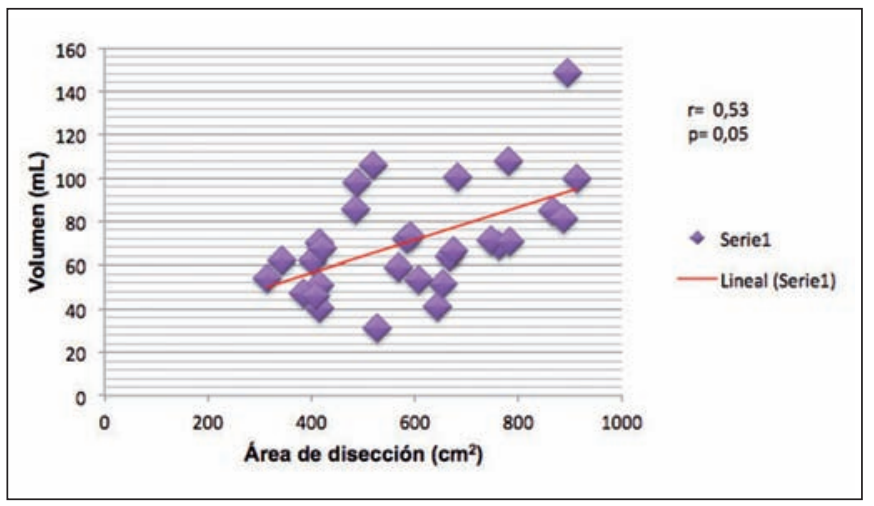

r: Coeficiente de Pearson.

entre los cirujanos plásticos, siendo considerada una medida postoperatoria correcta para evitar la colección de líquido en la pared abdominal.

Aunque no se han publicado investigaciones que concluyan el tiempo o el volumen ideal para retirar los drenajes aspirativos, está comúnmente aceptado que deben retirarse al $7^{\circ}$ día del postoperatorio o bien cuando el gasto sea menor de $30 \mathrm{ml}$; sin embargo, existen algunos autores que consideran inapropiado el uso prolongado o indiscriminado de los mismos, como Borile y col. ${ }^{(3)}$ quienes no consiguen diferencia estadísticamente significativa de la formación de seroma en pacientes en los que utilizaron drenaje en comparación con aquellos en los que solo emplearon faja postoperatoria, concluyendo además, que el uso prolongado de drenajes contribuye a aumentar la incidencia de infección local y de accidentes tromboembólicos.

En nuestra Unidad de Cirugía Plástica y Postbariátrica, a pesar de que compartimos esta última observación, enfrentamos una población de pacientes sometidos a regímenes de pérdida masiva de peso que presentan gasto elevado a través del drenaje postoperatorio tras dermolipectomía abdominal durante un tiempo superior a los 7 días, encontrando en nuestro estudio que el $10^{\circ}$ resultó ser el más frecuente para la retirada de los drenajes en nuestro grupo de estudio basándonos en la estabilización del gasto durante 48 horas y no en los conceptos empíricos conocidos; solo tuvimos 3 pacientes en los que la variación ascendente del volumen a través del drenaje ameritó otras medidas para la retirada del mismo, sin quedar demostrado que esta singularidad fuera causada por el uso prolongado de los drenajes.

La falta de consenso con respecto al manejo del drenaje postoperatorio para prevenir la formación de seroma ha motivado diversos estudios orientados a la evaluación de los factores que pueden condicionar la colección de fluido en la pared abdominal, así como las diversas medidas que se pueden emplear para prevenirla y para poder disminuir el tiempo que deben permanecer colocados los drenajes en el postoperatorio. Tal es el caso de Vinicius y col., ${ }^{(1)}$ quienes al comparar 3 grupos de pacientes reportaron un $57 \%$ de colecciones tras dermolipectomía en el grupo de pacientes en el que no emplearon drenajes frente a un $4 \%$ en el grupo en el que sí los utilizaron, concluyendo además que los grandes decolamientos, una hemostasia insuficiente, el uso abusivo del electrocauterio y el inicio de actividades de compresión abdominal inadecuadas, son factores predisponentes para la formación de estas colecciones. En este orden de ideas, Fang y col. ${ }^{(4)}$ y Costa-Ferreira y col., ${ }^{(5,6)}$ en 2010, se formularon la hipótesis de que al ser más superficial el plano de la elevación del colgajo abdominal, debería disminuir la necesidad postoperatoria de drenajes; llegando a la conclusión de que si se realiza la dermolipectomía en un plano más superficial, preservando la fascia de Scarpa, disminuye el volumen de gasto a través del drenaje, la formación de seroma y finalmente la necesidad de uso prolongado de los drenajes. Posteriormente a esta hipótesis, varios autores, como Friedman y col ${ }^{(7)}$ estudiaron histológicamente la arquitectura linfática de la pared abdominal, encontrando que los linfáticos son comunes en la dermis con una significativa proporción al igual que en las capas cercanas a la fascia de Scarpa y confirmando la presencia de canales linfáticos profundos, por lo que la preservación de la fascia de Scarpa en la región baja abdominal está asociada a la reducción de la tasa de formación de seroma en las abdominoplastias, siendo también esto comprobado por Tourani y col.$^{\left({ }^{8}\right)}$ en su estudio realizado en cadáveres.

Todas estas teorías han contribuido a estandarizar nuestra técnica para la realización de dermolipectomías abdominales, tanto para fines de esta investigación como en nuestra práctica habitual, en la que para lograr una hemostasia adecuada sin uso excesivo del electrocauterio utilizamos puntas de electro-bisturí no refractarias de cubierta de cerámica no conductiva. También realizamos la disección de los colgajos en un plano superior a la fascia de Scarpa, y empleamos de forma rutinaria drenajes aspirativos y prendas de compresión abdominal, ya que los pacientes con pérdida masiva de peso presentan una alta incidencia de formación de seromas. Diferimos por tanto con lo presentado por Quaba y col. ${ }^{(11)}$ quienes concluyen que al dejar la fascia de Scarpa resulta innecesario el uso de drenajes o suturas de tensión progresiva, puesto que no se producirá la formación de seromas. 
En nuestro protocolo, aunque no colocamos puntos de sutura desde el colgajo hacia la pared abdominal por creer que este método está relacionado con la formación de colecciones tabicadas y con un aumento del tiempo quirúrgico, opinión en la que coincidimos con Andrades y col., (2) sí empleamos drenajes aspirativos a pesar de preservar la fascia de Scarpa. Igualmente utilizamos prendas de compresión postoperatorias (faja y tabla de gomaespuma) en conjunto con el uso de drenajes de presión negativa, a diferencia de lo propuesto por Borile y col., ${ }^{(3)}$ en vista de que el inicio inadecuado de la compresión abdominal también se relaciona con la formación de seromas, tal como observan Vinicius y col. ${ }^{(1)}$

No obstante, estos estudios no consideran otros factores como el área de disección, que en pacientes no postbariátricos es menor en comparación con los pacientes de nuestra investigación, en los cuales medimos este dato durante la intervención quirúrgica, obteniendo un promedio de $582.98 \mathrm{~cm}^{2}$, y lo correlacionamos con el volumen obtenido a través del drenaje, tanto al inicio como en el momento de su retirada, encontrando una relación lineal moderada que explicaría que el área de disección influye en la cantidad de líquido que se obtiene a través del drenaje, pero que además existen otros factores que intervienen en su producción. Comprobamos así nuestra hipótesis con un nivel de confianza de $95 \%$.

De esta asociación también calculamos la cantidad de líquido que produce $1 \mathrm{~cm}^{2}$ de disección, que establecimos en $0.12 \mathrm{ml}$ con una $\mathrm{p}=0.05$. Este hallazgo es útil para poder inferir el drenaje esperado según la superficie de disección y poder a su vez predecir en el tiempo el momento más idóneo para retirar el drenaje.

En la actualidad no existen estudios para comparar nuestros hallazgos, por lo que creemos que son necesarias futuras investigaciones para comprobarlos. La única referencia que podría estar relacionada es el estudio de $\mathrm{Na}$ jera y col..$^{(14)}$ quienes reportan en su trabajo que el IMC es un factor independiente y significante en la formación de seromas después de una dermolipectomía abdominal, por lo que se supondría que a mayor IMC, mayor área de disección; pero en nuestro estudio el promedio de IMC estuvo en $27.85+5.49 \mathrm{~kg} / \mathrm{m}^{2}$, no siendo concluyente este dato.

Otro de los factores que consideramos en nuestra investigación fue la relación de los niveles de albúmina con la mayor producción de líquido, y a pesar de que en nuestro estudio los pacientes que presentaban niveles por debajo de $4 \mathrm{gr} / \mathrm{dl}$ mostraban un mayor promedio de líquido a través de los drenajes, esta asociación no fue estadísticamente significativa.

En base a todas estas observaciones, al realizar una abdominoplastia en pacientes que han sufrido pérdida masiva de peso recomendamos optimizar los parámetros bioquímicos, en especial los valores de albúmina y de proteínas totales por encima del rango normal. También mantener el drenaje durante al menos 10 días en el post- operatorio, tomando en consideración el área de disección practicada en cada paciente. Después del $10^{\circ}$ día del postoperatorio pinzar el drenaje en los casos en los que exista un aumento significativo del volumen del gasto, $y$ monitorizar la absorción del líquido a través de ultrasonido de partes blandas en la pared abdominal en el momento del pinzamiento y 24 horas más tarde, para decidir su retirada. Finalmente, retirar el drenaje cuando se observe un volumen estable de gasto a través del mismo durante 48 horas, independientemente de la cantidad.

\section{Conclusiones}

Existen diversos factores que influyen en la formación de líquido tras una dermolipectomía abdominal, y en poblaciones especiales como es el caso de los pacientes con pérdida masiva de peso, el aspecto bioquímico, específicamente los valores de proteínas totales y albúmina, se asocian con la variación del volumen de gasto a través de los drenajes en comparación con la población general. Sin embargo, nuestra investigación no conseguió una relación estadísticamente significativa que logre explicar el fenómeno, considerando que toda la muestra tenía valores dentro del rango normal.

Otro aspecto diferente en estos pacientes es el que dada su morfología, a pesar de presentar IMC cercanos a la normalidad, el área de disección en la dermolipectomía abdominal es amplia, y al relacionar dicho parámetro con el volumen de líquido obtenido a través de los drenajes postoperatorios en distintos momentos de la investigación, conseguimos una correlación lineal moderada pero estadísticamente significativa entre el área de disección y el gasto a través del drenaje, lo que podría explicar la mayor producción de líquido observada en estos pacientes así como la necesidad de que la retirada de los drenajes en ellos se realice más tardíamente.

Finalmente, establecimos una relación lineal moderada entre la cantidad de líquido que se produce y el área de superficie decolada, dato que podría ser predictor del volumen obtenido en 24 horas a través del drenaje según el área disecada; y al observar la evolución del gasto durante los primeros 10 días de postoperatorio, podremos decidir la retirada del drenaje o evaluar la necesidad de su pinzamiento cuando se evidencie que hay un aumento del patrón de gasto.

\section{Dirección del autor}

Dra. Linda Lorena Rincón Rubio

Unidad de Cirugía Plástica y Postbariátrica

Centro Diagnóstico Docente Las Mercedes

Apartado postal 90314

El Hatillo, Caracas 1080, Venezuela

Correo electrónico: plasticabariatrica@gmail.com 


\section{Bibliografía}

1. Vinicius M, Mendonça L, da Motta Martins P. Incidência de Hematoma e Seroma em Abdominoplastia com e sem uso de Drenos. Rev. Soe. Bras. Cir. Plást. São Paulo 2002; 17 (1): 69-74.

2. Andrades P, Prado A, Danilla R. Progressive Tension Sutures in the Prevention of Postabdominoplasty Seroma: A Prospective, Randomized, Double-Blind Clinical Tria. Plast. Reconst. Surg. 2007; 120(4): 935-946.

3. Borile G, Pavelecini M, Dreher R. The Use of Suction Drains in Abdominal Dermolipectomy: A Randomized Clinical Trial. Plast. Reconst. Sur.g 2008; 121(4): 228e-229e.

4. Fang R, Lin S, Mustoe, T. Abdominoplasty Flap Elevation in a More Superficial Plane: Decreasing the Need for Drains. Plast. Reconst. Surg. 2010;125(2): 677-682.

5. Costa-Ferreira A, Rebelo $M$, Vásconez $L$, et al. Scarpa Fascia Preservation during Abdominoplasty: A Prospective Study. Plast. Reconst. Surg. 2010; 125(4):1232-1239.

6. Costa-Ferreira A, Rebelo M, Silva A, et al. Scarpa Fascia Preservation during Abdominoplasty: Randomized Clinical Study of Efficacy and Safety. Plast. Reconst. Surg. 2013. 131(3): 644651.

7. Friedman T, Coon D, Kanbour-Shakir A, et al. Defining the Lymphatic System of the Anterior Abdominal Wall: An Anatomical Study. Plast. Reconst. Surg. 2015. 135(4): 1027-1032.
8. Tourani S, Taylor G, Ashton M. Scarpa Fascia Preservation in Abdominoplasty: Does It Preserve the Lymphatics? Plast. Reconst. Surg. 2015. 136(2): 258-262.

9. Moretti E, Gómez García F, Monti J, Vásquez G. Investigación de seromas postliposucción y dermolipectomía abdominal. Cir plást iberolatinoam. 2006; 32(3):151-160.

10. Llanos S, Macan F, Llanos P, Llanos F. Incidencia de seroma postoperatorio tras reparación de la fascia de Scarpa en abdominoplastia-liposucción. Cir plást iberolatinoam. 2013; 39(2) 129-136.

11. Quaba A, Conlin S, Quaba, O. The No-Drain, No-Quilt Abdomin.oplasty: A Single-Surgeon Series of 271 Patients. Plast. Reconst. Surg 2015; 135(3): 751-760.

12. Di Martino M, Nahas F, Kimura A, Sallum N, Ferreira L. Natural Evolution of Seroma in Abdominoplasty. Plast. Re const. Surg. 2015; 135 (4): 691e-698e.

13. Shermak M, Rotellini-Coltvet $L$, Chang D. Seroma Development following Body Contouring Surgery for Massive Weight Loss: Patient Risk Factors and Treatment Strategies. Plast. Reconst. Surg. 2008; 122 (1): 280-288.

14. Najera R, Asheld W, Sayeed S, Glickman L. Comparison of Seroma Formation following Abdominoplasty with or without Liposuction. Plast. Reconst. Surg. 2011; 127 (1): 417-422. 